\title{
The Effect of Inaccurate Traffic Data for Ramp Metering: Comparing Loop Detectors and Cameras Using Information Utility
}

\author{
Gerdien A. Klunder*, Henk Taale**, Leon Kester, *** \\ Serge Hoogendoorn**** \\ *Delft University of Technology, TNO, Smart Mobility, PO Box 49, 2600 AA \\ Delft, The Netherlands (Tel: +31(0)888668468; e-mail g.a.klunder_1@tudelft.nl). \\ ** Transportation and Planning Department, Delft University of Technology, P.O. . Box 5048, 2600 GA Delft \\ (Tel: +31887982498; e-mail h.taale@tudelft.nl) \\ *** TNO, PO Box 96864, 2509 JG Den Haag, \\ The Netherlands (Tel:+31888664067; e-mail leon.kester@tno.nl) \\ **** Transportation and Planning Department, Delft University of Technology, P.O. Box 5048, 2600 GA Delft \\ (Tel: +31152785475; e-mail s.p.hoogendoorn@tudelft.nl)
}

\begin{abstract}
This paper introduces a topic which is of growing interest in the traffic control and management community, namely the relation between traffic data quality and the efficiency of traffic management. After some explanatory background and state of the art, it will become clear that no standard approach is available for traffic engineers to determine which accuracy of traffic data is needed for a certain traffic management application. In this paper we will show how the effect of inaccurate measurements on network level can be quantified by determining information utility functions. This approach is illustrated by presenting the results of a simulation study into the effect of inaccurate loop detector measurements on a ramp metering system. The relation between different accuracies of flow and speed measurements as input for a ramp metering system and the corresponding effect on the network performance is studied with a micro simulation model. The results of the simulation are used in a cost-benefit analysis for a case comparing loop detectors with camera measurements, where loop detectors are considered more accurate but also more expensive. We will show that higher measurement accuracy will lead to a better performance of the ramp metering system, resulting in a higher average network speed, and also quantify this relationship.
\end{abstract}

Keywords: Data Quality, Network Performance, Utility Functions, Ramp Metering, Micro simulation, Traffic Data, Traffic Management

\section{INTRODUCTION}

More and more data is coming available in the recent years, e.g. traffic data, GPS data, weather and air quality data, behavioural data and financial data. In a study from IBM (2013) it was stated that $90 \%$ of the data in the world of today (2011) has been created in the last two years before that. As a consequence, in just a few years the challenge has shifted from 'if we only had the data' to 'how can we drive better intelligence from the data' (K.T. Parker, President and CEO VIA Metropolitan Transit, unpublished). The increase in data also applies to traffic. Not only more data is coming available, but also different types of data from different sources, such as induction loop detectors, floating car data (FCD), GPS or GSM data, blue tooth data etc. Especially, floating car data are a promising and growing data source, fed by the recent growth of smartphones and smartphone GPS applications. However, many of these data are not publicly available, because navigation system providers collect data via their navigation systems but keep these within their own company to generate travel time information for their users or to commercially sell the data.
Dynamic traffic management and information is used by road operators in order to improve network utilization, safety or the environment. An example of this is managing the traffic flow by influencing speeds, lane use, route choice or merging operations. Several measures can be used for that, for example variable message signs (VMS), Dynamic Route Information Panels (DRIPs), ramp metering, etc. In order to operate the measures, to generate traffic information and to choose the best suitable measure, traffic data are required.

Each data type has its own characteristics, semantics, and quality. There are many dimensions of traffic data quality, such as spatial coverage, temporal aggregation, timeliness, and reliability. What these dimensions have in common is accuracy. For example, a communication delay will usually lead to less accurate real-time data. In this paper, while it can be regarded as a general approach to investigate the relation between data quality and performance, we will focus on accuracy. The required quality for a dynamic traffic management (DTM) measure or traffic information service differs, depending on the type of measure or information needed, but little is known about the precise quality needed or the relation between quality and performance of the measure. Some measures are more time critical than others, while also 
the required accuracy requirements may differ. However, substantive research to establish requirements for the quality of traffic data in relation to the intended traffic management goals is lacking, while more and more new traffic data are coming available and the demand for reliable traffic information is increasing. Therefore more research on this subject is needed.

If the requirements for traffic data can be determined for certain traffic management applications, this will give new possibilities for better traffic management. It may lead to a better achievement of the traffic management goals with the same data, i.e. more efficient data use, for example by applying dedicated algorithms suitable for the available data with the given data quality. Also, better requirements for data acquisition can be imposed to traffic data providers, which may lead to cost reduction when less detailed/accurate data are sufficient, or when data acquisition can be improved for better results, for example by choosing optimal monitoring locations. Clearly, since resources are limited, one should use them for traffic management where and when the need is highest.

In this paper, the relation between different accuracies of flow and speed measurements as input for a ramp metering system and the corresponding effect on the network performance is studied. This is illustrated with a simple case comparing loop detectors with camera measurements, where loop detectors are more expensive but also more accurate. We will show that higher measurement accuracy will lead to a better performance of the ramp metering system, resulting in a higher average network speed, and we will quantify this relationship with an information utility function. This will allow us to perform a design-time optimisation, i.e. determining optimal design choices of the system before the actual operation. By monetising the delay time of traffic, a cost-benefit decision will be made for either cameras or loop detectors. This approach can be extended with utility based reasoning for run-time adaptive data processing (algorithm selection, parameter tuning, processing techniques and when and what to communicate), e.g. deciding real-time which data should be communicated, taking into account communication costs or other restrictions, as explained in Foeken et al. (2009). As such, the performance of traffic management applications can be dynamically improved.

\section{BACKGROUND}

In this paragraph, we will first give a state of the art, in which we will consider several relevant topics for the explained problem. First, we investigate existing studies on the topic, which proofs the relevance in this relatively new topic. Then we look at the effect of fusing different types of data for the data quality needed. Finally we explain that also organizational aspects are relevant for this problem. Next, we explain how errors may be amplified or reduced on the path from measurement to the final message to the road users through the data processing chain. This all will lead to the conclusions that no standard approach is available yet to quantify the relation between data accuracy and the efficiency of traffic management, and it will also lead to a first proposal how one can approach this, using utility theory and sensitivity analysis, as explained in the next paragraph.

\subsection{State-of-the-Art of research on traffic data quality}

Klunder et al. (2012) performed a preliminary study on the relation between inaccurate traffic data and route choice, which concluded that accurate traffic counts are important for route choice information in case both route alternatives are close to oversaturation. Tampère et al. (2011) investigated the relation between data quality and dynamic traffic management. However, this research studied only the effect on the resulting information or traffic management measure, not the impact on the traffic system itself, and they concluded that more thorough research is needed on this. Also at European level it has been identified that there is a lack of common quality criteria for traffic data and services. The QUANTIS project (Öörni et al., 2010) aimed to provide preliminary insights into the issue. In the U.S. it is recognized that the matter of data quality has become more urgent in recent years by the increase of ITS applications and various travel information systems, as reported in the "Data Quality White Paper" from the Federal Highway Administration (Ahn et al., 2008).

In order to determine traffic data in practice, a comparing sensing system is needed. Though, all sensors have to cope with inaccuracies, such that a perfect 'ground-truth' dataset will generally not exist. Depending on the purpose, also nonperfect data or combinations of all available data can be used as a reference. Since fusing different types of data influences the minimum quality level needed, for this problem it is also relevant to study the effect of combinations of these different types of data. Concerning the use and comparison of induction loop data or FCD data, research had been done already for example by Gazis and Knapp (1971). In this article, a new method is put forward for fusing heterogeneous and semantically different data from different traffic sensors. Van Lint and Hoogendoorn (2007) compared and used both induction loop data and FCD for traffic state estimation, and also looked at the economic benefits by performing a costbenefit estimation.

An important development concerning collection and distribution of traffic data in the Netherlands is the National Data Warehouse for Traffic Information (NDW) (Viti et al., 2008). The NDW is a partnership of 15 Dutch authorities that joined their strengths to provide complete, reliable and up-tothe-minute information on the status of the main Dutch road network. The NDW is the Dutch databank that collects, processes, stores and distributes all relevant traffic data. Quality requirements have been defined by the NDW and imposed to traffic data suppliers. These quality requirements are not differentiated for traffic management applications. Currently, there are discussions about redefining the quality requirements, especially to differentiate them, because the current quality requirements cannot always be met and will lead to high costs, as presented by Felici and Vroom (2012).

Furthermore, the current operating traffic management systems, have been developed many years ago (Klijnhout, 1984) and in the meantime the systems and algorithms have evolved to such a complexity that it is not easy to switch to 
another (more efficient) system. When the current situation would be totally blank without any monitoring system, one could design a much more efficient traffic management system than the current one. In order to make this switch now, high initial costs are needed and many organizational issues will need to be solved. As such, the Netherlands has to deal with the law of the handicap of a head start, being one of the countries with the most extensive and oldest traffic monitoring systems. This makes it more difficult to change the current system, however, considering the current economic situation which asks for budget cuts, the time is ripe to investigate alternative solutions utilising the benefits of new data.

\subsection{Traffic data processing chain}

In this paragraph it will be explained how inaccurate input data may lead to a change in the traffic system and how errors may be amplified or reduced on the path from measurement to the final message on the road. The process from traffic data to the end user consists of a chain of several steps. In each step, existing errors may be enlarged or reduced. In order to determine the effect at the end of the chain, each step should be analysed.

It starts with the processing of the raw data. This includes some basic improvements such as complementing missing data and removal of outliers. It may also consist of advanced processing techniques for combining different data sources (e.g. Kalman filtering), and prediction of the future traffic state with prediction or simulation models. These processing and filtering techniques are meant to improve the quality of the data and to reduce the initial error.

After the processing of the data, the next step is to use the data for service provision or traffic management, i.e. showing travel times on DRIPs. In this step, usually algorithms and/or models are used to translate the traffic data into the required output of the service. It depends on the nature of the measure and the algorithm if it is sensitive for small errors on the input data. For example, if a boundary value is used to switch a measure on or off, it is only sensitive for variations around this boundary value. Also timing can be an issue for data quality. For example, when travel times are measured at the end of a trajectory, these travel times are already outdated and will not hold for the vehicles starting now. For very time critical applications such as queue tail warning or ACC, even the calculations of the model might become a bottleneck for the actuality of the processed data.

Finally, the information or traffic management measure will be presented to the end user, which might change its behaviour and therefore have an impact on the resulting traffic system. The aim of traffic management is generally to improve the traffic performance, but when the traffic management measure is controlled by erroneous traffic data, the effect might be adverse. When drivers comply to a traffic management measure which is based on an incorrect traffic state estimation, this will not have the intended effect (e.g. when drivers are advised to follow an alternative route, while this route turns out to suffer from congestion). The whole process can be summarized in a scheme as shown in Fig. 1.
Quality of data is furthermore highly related to costs for data acquisition and processing. Higher quality can be obtained with using more or more accurate monitoring instruments. It is not clear beforehand how higher costs for data acquisition relate to higher service quality at the end of the chain. So in the end, it is a matter of finding the best combination of costs and benefits.

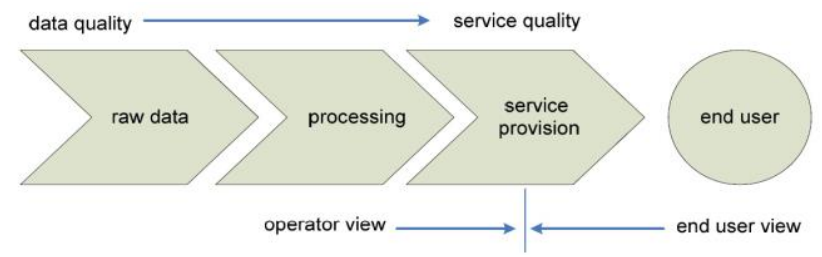

Fig. 1. Flow scheme from raw data to the end user.

\section{UTILITY THEORY AND SENSITIVITY ANALYSIS}

From the above, it becomes clear that the problem of determining which accuracy of traffic data is needed for a certain traffic management application is very relevant, however, no standard approach is available for traffic engineers to do this. In this paper we will propose an approach based on information utility functions for this problem.

The effect of inaccurate traffic measurements on traffic management can be analysed using utility theory and sensitivity analysis, the study of how the uncertainty in the output of a mathematical model or system (numerical or otherwise) can be apportioned to different sources of uncertainty in its inputs (Saltelli et al., 2008).

Utility theory has for example been applied in the military domain (e.g. Ditzel et al., 2013), while in traffic engineering the application of utility theory for analysing the effect of inaccurate traffic data seems unknown. In order to value new information, the utility function tells how utile new information is. It can be defined as the sensitivity of a certain parameter with regard to the value of the measure. The utility function quantifies the utility as a function of the important features of information, as explained in Foeken and Kester (2012). The value of sensor information is defined as the gain in utility that information brings to the current awareness (of the traffic state), where utility is calculated by an information-utility function. This information-utility function reflects the increase in effectiveness of the (control) system due to an increase in quality of the information.. Next, one should determine the value of the result of the measure, which should be related to the traffic management goal. Usually for traffic management this is something like the average network speed or minimizing the amount of congestion. For the ramp metering case, the utility function will express the sensitivity for inaccurate speed and flow measurements on the mean network speed. The value can be monetized (e.g. by using value of time) in order to compare it with the costs of the measure. The difference between the Value and the Cost is called the Reward. Analogous to decision theory, the optimal decision is taken based on the maximum reward. 
In order to quantify the effect of uncertainty of a certain input parameter on the final performance of the system, first a definition is needed of the objective of the measure and how this can be measured and quantified. Then the Measure of Performance (MOP) can be formulated. In the end, a quantitative relationship should be found between the accuracy of the input variables and the MOP.

There are different types of errors possible for input values, such as missing values, outliers, biases and random errors. In this paper, we focus on biases and random errors. A bias is a fixed deviation from the real value, while random errors can have all kinds of distributions. Most errors can be modelled by a combination of a fixed bias and a random part with mean 0 and a certain standard deviation. In order to test the sensitivity for each of these, they should be separated in the analysis.

A utility function can be derived analytically if the whole chain as shown in Fig. 1 can be described with mathematical functions. For the processing of the raw data and the algorithm of the traffic management measure, this might be possible (though this is often non-linear and not continuous), however, this is much more difficult or unknown for the driver behaviour and the result on the traffic flow. Therefore a good alternative approach is to use simulation. The simulation environment will allow to derive perfect measurements while also input data can be adapted in order to mimic real measurements with a certain error. The adapted (inaccurate) measurements from the simulation should be used as input to the traffic management measure in the simulation. The performance can be measured based on the perfect measurements from the simulation. For each variation of the accuracy of a certain input parameter, a new simulation run should be done with equal settings except for the accuracy of that measurement value.

While in this example only design time optimisation is applied ('should we install camera's or loop detectors'), these concepts can also be applied for runtime optimisation using utility based reasoning, as explained in the Introduction. A good example where these concepts have been worked out to select the optimal strategy based on inaccurate measurements in the military domain can be found in Ditzel et al. (2013).

\section{RAMP METERING CASE STUDY}

We will illustrate the workings of the approach by means of a ramp-metering case study. Ramp metering is a traffic signal control system placed at onramps with the purpose to prevent congestion on the motorway by restricting the inflow of vehicles onto the motorway via the onramp. The length of the total cycle determines the inflow in vehicles per hour to the motorway. The green time needs to be tuned such that each green period, only one vehicle can go through; the so-called "one car per green" strategy. At some locations, also a "two cars per green" strategy is used. In The Netherlands the green time is dynamic, depending on the acceleration of vehicles.

Ramp metering is an accepted traffic management measure in The Netherlands. At the end of 2005 in The Netherlands 54 on-ramps had a ramp metering system (Middelham and Taale, 2006). Today there are more than 100 on-ramps equipped with a metering system. In several assessment studies, effects of a capacity increase ranging from $1 \%$ to $5 \%$ were found, as well as a speed increase on the motorway from 4 to $30 \mathrm{~km} / \mathrm{h}$.

\subsection{Loop detectors versus cameras}

Suppose a road manager has decided to apply ramp metering, but he has no measuring equipment yet. He can choose to install inductive loop detectors or cameras. The loop detectors have a higher accuracy but are also more expensive to purchase, install and maintain. The following case study shows how a sensitivity analysis and utility-based optimization can be used in order to make the most costeffective decision. For calculating costs of loop detectors we use numbers from Dutch practice based on personal correspondence with Rijkswaterstaat (part of the Dutch Ministry of Infrastructure and Environment). The costs for loop detectors for the signalling system (MTM) are: replacement costs $€ 1.548$ per pair $+€ 9.502$,- per detector station (one for several loop detectors), total maintenance costs $€ 633$,- per year per detector, lifetime 15 years. For a video camera we assume a budget type camera: replacement costs $€ 3.000$, total maintenance costs $€ 1.000$,- per year, lifetime 10 years. However, accuracy of camera measurements is usually less than for loop detectors. For the ramp metering algorithm at a two-lane motorway, usually at least four detector pairs are used, (pairs because also the speed should be measured), one pair at the motorway at each lane, one at the onramp after the metering signal. Also, upstream of the traffic signal at the onramp, usually detectors are installed which can be used to monitor the queue before the signal. In case the queue gets too long (e.g. blocking back effect when blocking an upstream intersection), the ramp metering can be switched off. In our scenario, where we consider a two-lane motorway with a one-lane onramp, the considered number of needed loop detectors is therefore 8 ( 2 pairs on the motorway and 2 pairs on the onramp) with 3 detector stations (one at the motorway and two at the onramp). In Dutch practice, even more loop detectors are installed to accommodate ramp metering. A typical detector layout contains 7 detectors on the onramp, as can be seen in Middelham and Taale (2006). A camera can monitor several lanes, though in that case the accuracy will be less. For our case we consider 3 cameras (one at the motorway covering two lanes and two at the onramp, one downstream of the traffic light and one further upstream to monitor spillback).

The question now is which is the most cost effective solution, loop detectors or cameras? This may depend on the time horizon: initial costs of loop detectors are higher, but might be earned back after several years due to better congestion prevention.

\subsection{Ramp Metering Algorithm}

In this study, a feedback ramp metering algorithm developed by Papageorgiou et al. (1991) is used, called ALINEA, which is one of the most well-known and widely applied ramp metering algorithms. The algorithm is defined as follows:

$$
q_{\text {cont }}(k)=q_{\text {cont }}(k-1)+K_{r} \cdot\left[o_{\text {crit }}-o_{\text {out }}(k)\right]
$$


where $q_{\text {cont }}$ is the onramp flow after the ramp metering control, which can either be calculated or measured. The occupancy is measured at the motorway downstream of the onramp. The algorithm tries to keep the occupancy at a certain critical (desired) occupancy. Since the output of the system is used as input to the algorithm, it is a closed-loop system, which is generally more stable than an open system.

Though the algorithm is occupancy based, in practice in the Netherlands it is mostly applied with speed and flow as input, since the recent loop detectors do not give occupancy as output. In our simulations the algorithm is therefore applied with the density (vehicles per kilometre, calculated as flow divided by the speed) instead of occupancy. The density is smoothed by applying the filter developed by Treiber (2002). This prevents too many fluctuations (on- and off switching and ramp metering flow). The weighting factor $K_{r}$ has to be adapted for this case. Normally a value of 70 is chosen, this was adapted to 10 . This setting is rather arbitrary but was chosen such that the difference between measured and desired density will lead to a reasonable difference in the ramp metering flow per control interval.

The ramp metering system is activated when a certain density boundary is exceeded, and turned off when the density drops below a lower density boundary. The algorithm can be overruled in practice, for example when the queue upstream of the ramp metering signal spills back too much on the urban network.

\section{SIMULATION EXPERIMENT}

We performed a simulation experiment in Vissim of a ramp metering control system to investigate the effect of inaccurate speed and flow data on the network performance. The ALINEA algorithm has recently been tested in simulation for the A10 west of Amsterdam in the so-called 'Praktijkproef Amsterdam (PPA)' or Field Operational Test Integrated Network Management Amsterdam (Hoogendoorn, 2013). For this purpose, a Vissim simulation has been set-up for the A10 West, calibrated visually until the model showed realistic traffic flow behaviour. For the current study, the same simulation set-up and implementation of ALINEA have been used. It should be noted that a more exact calibration was not needed for this study, because it serves as an illustration of the type of analysis proposed in this paper, for which the exact outcomes in numbers are less important.

The network consists of a motorway stretch with 2 lanes of 4 kilometres with two off ramps and two on-ramps with (coordinated) ramp metering systems. The total demand on the motorway is 3067 during one hour, with a peak demand of $3500 \mathrm{veh} / \mathrm{h}$, added with 750 vehicles/hour on the first onramp and 600 vehicles per hour on the second onramp. This represents a (morning) peak hour. In order to be able to study the effect of inaccurate loop detector without distortion effects of the coordinated ramp metering, only one ramp metering control system was used on the first onramp. The parameters of the ALINEA algorithm were chosen as follows: critical density $\left(k_{\text {crit }}\right) 30 * 0.8 \mathrm{veh} / \mathrm{km} /$ lane (scaling with 0.8 to stay below the critical density), $K_{r}=10$. The resulting metering flow is bounded between 150 and 1500 .
The ramp metering control will become active when the density exceeds $25 \mathrm{veh} / \mathrm{km} / \mathrm{lane}$, and will become inactive when the density gets below $20 \mathrm{veh} / \mathrm{km} / \mathrm{lane}$. This setting has been chosen based on expert judgment and has not been optimised on the ramp metering performance.

\subsection{Experiments}

Both the speed and the flows have been artificially adapted by adding noise or a bias to it, in order to simulate inaccurate measurements. Two types of errors have been investigated: a fixed error (bias) of a certain size, varying from $-40 \%$ to $40 \%$ of the real value with steps of $1 \%$, and a normally distributed random error with mean 0 and standard deviation varying in size from 0 to $50 \%$ with steps of $0.05 \%$.

For each setting (error of two types and the above given different sizes on speed or flow), a full Vissim simulation has been performed of one hour. Each simulation has been done with the same seed in order to have comparability between the different simulations.

Several measures of performance have been derived from the simulation to represent the effect on network level. In this paper, we only consider the average network speed (total average of both the main road and the ramps).

\subsection{Results}

The results are shown in Fig. $2 \mathrm{t} / \mathrm{m} 5$. In these figures, each dot is the result of a full simulation run. The results between the runs show a high variation, even while the same seed has been used. This is the consequence of changes in the ramp metering and the stochasticity in the simulation: even a small change in the ramp metering operation can cause a very different traffic flow. The results are fitted to polynomial equations, which give a reasonably good fit, except for figure 5 for which the fit is poor according to the $r^{2}$ statistic. Without ramp metering, the average network speed (including the ramps) is $47.4 \mathrm{~km} / \mathrm{h}$. With ramp metering, this is improved up to $55 \mathrm{~km} / \mathrm{h}$. With a random error of $50 \%$ this goes down to approximately $49 \mathrm{~km} / \mathrm{h}$, still a little better than the case without ramp metering.

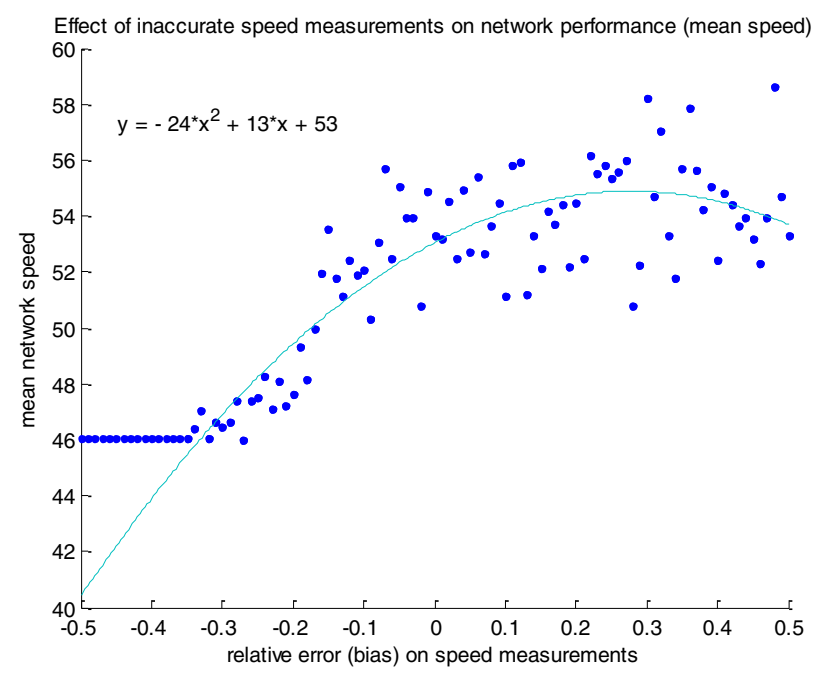

Fig. 2 Effect of a fixed error of the measured speeds on the mean network speed $\left(r^{2}=0.84\right)$. 


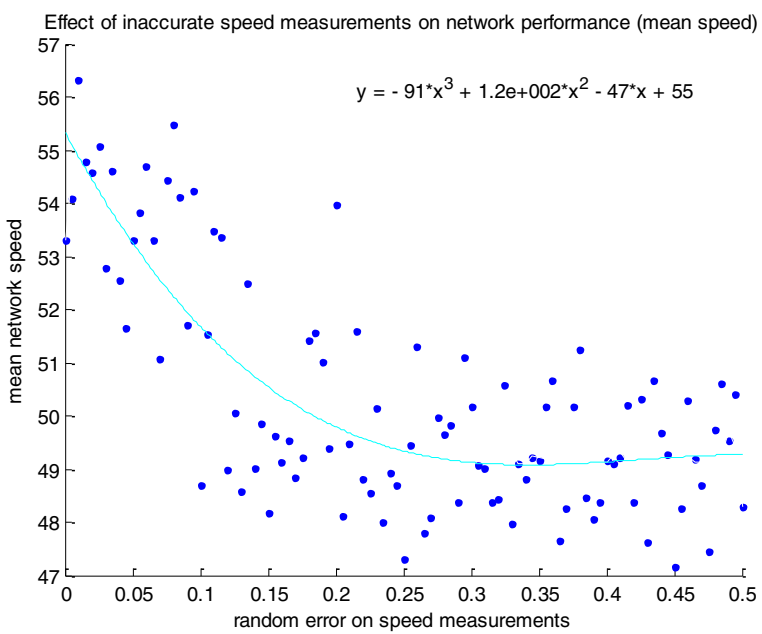

Fig. 3 Effect of varying standard deviation of measured speeds on the mean network speed $\left(r^{2}=0.61\right)$.

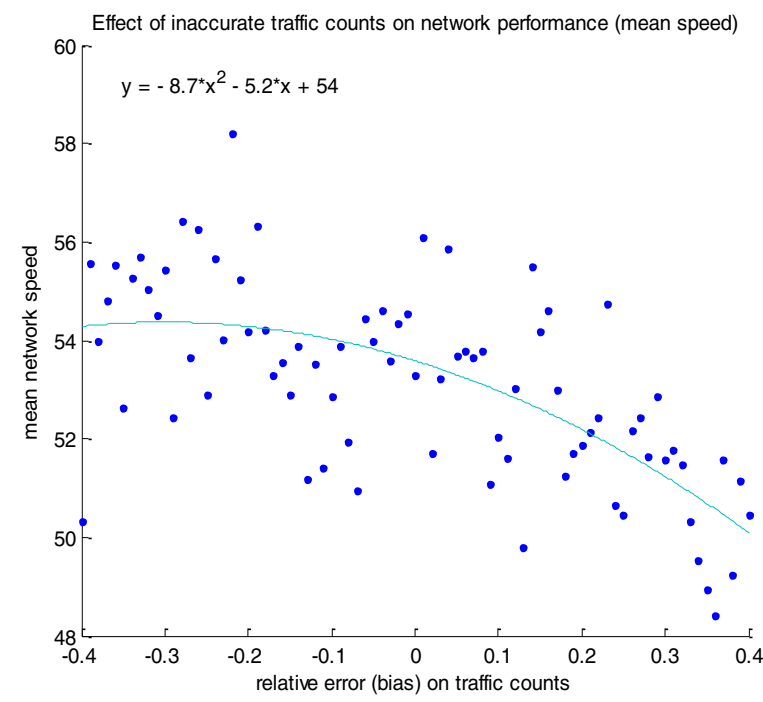

Fig. 4 Effect of a fixed error of the measured traffic counts on the mean network speed $\left(r^{2}=0.44\right)$.

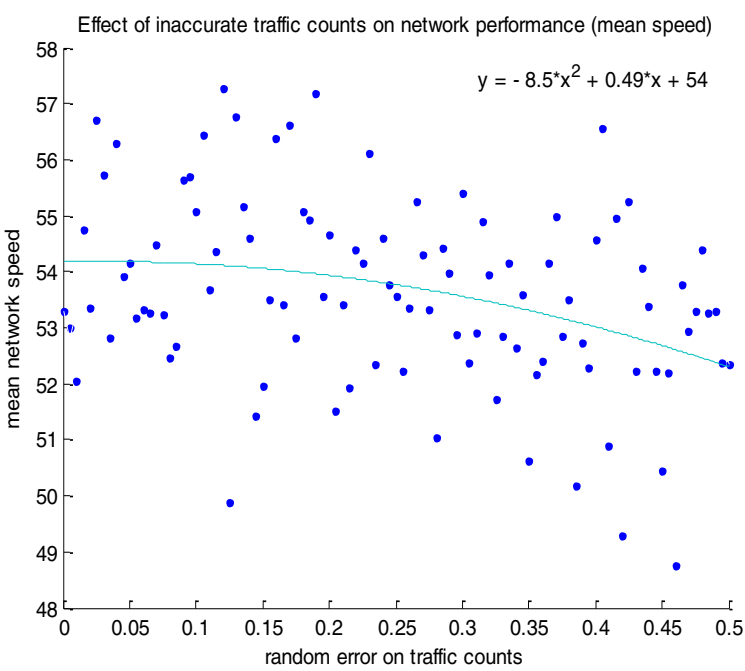

Fig. 5 Effect of varying standard deviation of the measured traffic counts on the mean network speed $\left(r^{2}=0.13\right)$.
Comparing Fig. 2 and 3 with Fig. 4 and 5, it appears that there is a larger effect of inaccuracies on speeds than inaccuracies on traffic counts. This is probably due to the fact that dividing by the speed has a large influence on the density. A positive error (bias) on speed initially improves the network performance. Apparently the base case is not tuned optimally, such that the system works better with overestimated speeds. From the fitted $2^{\text {nd }}$ order polynomial we can easily calculate that the optimum is achieved for $27 \%$ overestimation. In Fig. 2, for a speed bias $<-30 \%$ there is no further degradation of the network performance (the speed remains the same), which can be explained since in that case the ramp metering system will then be turned on continuously with minimum ramp metering flow (minimum green time) which will be the same for even lower measured speeds.

The difference in effect for inaccuracies on speeds and flows can be explained as follows. The ramp metering system will work less effectively when it will not turn on while there is a high density on the motorway, or when it will turn on while there is no high density on the motorway. An incorrectly too low occupancy will be measured when the flow is measured too low or the speed is measured too high, since the density is calculated as flow divided by the speed. Therefore, an underestimation of the speed has a larger effect on the calculated density than an underestimation of the flow, and also the effect is opposite.

Another important part of the algorithm that influences the operation is the on- and off switching. An estimated too high or too low density will activate the turning on and off of the ramp metering incorrectly.

\subsection{Cost-benefit analysis of loops versus cameras}

Coming back to our illustrative case where we compare loop detectors with a higher accuracy to cameras, we can determine with some simple calculations based on the results above which would be the most cost-effective investment choice.

Let's assume that both systems do not have a bias in the speed measurements, but the loop detectors have a random speed error of $5 \%$ (also based on personal communication with Rijkswaterstaat) and the cameras have a larger random error of $8 \%$. Considering the flow, both monitoring systems tend to underestimate the flow because they can 'miss' vehicles. For the loop detectors we take a bias on the flow of $-2 \%$ and a random error of $5 \%$, whereas for the cameras we take a bias on the flow of $-10 \%$ and a random error of $10 \%$. Now, assuming these errors are independent we may sum up the effects (with regard to the base case without errors) using the fitted utility functions. The results are given in Table 1, with the effects on the network speed in brackets. The reference case shows the situation without ramp metering, the base case the situation with ramp metering without measurement errors. The cameras only perform slightly better than the situation without ramp metering. These effects seem small, however, scaling it up to a yearly level still leads to a large total delay. Considering the network length of 4 kilometres and the total vehicle demand of 4417 vehicles in the simulation, during a one hour peak two times a day for all 
working days in a year, this accounts for an extra delay of 7241 vehicle hours per year for the inaccuracy of loop detectors (compared to the base case without errors), and 14745 vehicle hours extra delay per year for the inaccuracy of cameras.

Table 1. Scenarios with Average Network Speed Change With Regard to the Base Case

\begin{tabular}{|c|c|c|c|c|c|c|}
\hline & \multicolumn{2}{|c|}{ Speed error } & \multicolumn{2}{|c|}{ Flow error } & \multirow{2}{*}{$\begin{array}{l}\text { Netw. } \\
\text { speed } \\
\text { change } \\
(\mathrm{km} / \mathrm{h})\end{array}$} & \multirow{2}{*}{$\begin{array}{c}\text { Average } \\
\text { netw. } \\
\text { speed } \\
(\mathrm{km} / \mathrm{h})\end{array}$} \\
\hline & Bias & Rand & Bias & Rand & & \\
\hline Reference & 0 & 0 & 0 & 0 & -5.9 & 47.4 \\
\hline Base & 0 & 0 & 0 & 0 & 0.0 & 53.3 \\
\hline $\begin{array}{l}\text { Loop } \\
\text { detectors }\end{array}$ & 0 & $\begin{array}{r}5 \% \\
(-2.1) \\
\end{array}$ & $\begin{array}{r}2 \% \\
(-0.1) \\
\end{array}$ & $\begin{array}{r}5 \% \\
(0.0) \\
\end{array}$ & -2.2 & 51.1 \\
\hline Cameras & 0 & $\begin{array}{r}8 \% \\
(-3.0)\end{array}$ & $\begin{array}{r}10 \% \\
(-0.6)\end{array}$ & $\begin{array}{r}10 \% \\
(-0.0)\end{array}$ & -3.6 & 49.7 \\
\hline
\end{tabular}

Calculating with a Value of Time of about $€ 11$,- per hour (Warffemius, 2013), this accounts for approximately $€$ 82.544 ,- per year extra costs for the cameras compared with the loops. Calculating the gain with regard to the reference case, this leads to a reduction of 14034 vehicle hours delay per year for loop detectors and of 8970 vehicle hours delay per year for the cameras.

Finally we can calculate what will be the most cost effective solution on the longer term, comparing loop detectors with cameras. In accordance with the utility theory discussed in chapter 3 a general cost equation for this problem can be formulated as follows:

$c_{\text {total }}(t)=c_{\text {repl }} \cdot n_{\text {det }}+c_{\text {maint }} \cdot n_{\text {det }} \cdot t$

with $c_{\text {repl }}$ the replacement costs per detector, $c_{\text {maint }}$ the maintenance costs per detector per year, $n_{\text {det }}$ the number of detector stations and $t$ the number of years since instalment.

For a ramp metering configuration as introduced in paragraph 4.1 with 8 loop detectors ( 4 pairs) and 3 detector stations this gives a cost function of

$c_{\text {total_det }}(t)=1548 \cdot 4+3 \cdot 9502+t \cdot 633 \cdot 8$

Similarly, a benefit or value function can be defined as:

$V(t)=V o T \cdot$ delay $_{\text {red }} \cdot t$

with VoT the average Value of Time per hour delay, and delay $_{\text {red }}$ the reduction in the delay in vehicle hours per year.

Finally, balancing cost and benefits the reward function can be defined as:

$R(t)=\operatorname{VoT} \cdot$ delay $_{\text {red }} \cdot t-c_{\text {total }}(t)$

The total Reward after $t$ years for the loop detector configuration is then:

$$
R_{\text {det }}(t)=11 \cdot 14034 t-(34698+t \cdot 5064)
$$

Cameras: 3 cameras needed. Total costs after $t$ years:

$$
c_{\text {total_cam }}(t)=3 \cdot(3000+t \cdot 1000)
$$

Total Reward after $t$ years:
$R_{\text {cam }}(t)=11 \cdot 8970 t-3 \cdot(3000+t \cdot 1000)$

Visualizing these equations gives the following Fig. 6.

This figure shows that for our case, already within one year it is more cost effective to use loop detectors than cameras. However, a camera has additional possibilities with regard to loop detectors, namely that cameras are capable to view a trajectory of the road instead of a fixed point measurement. With this, for example more accurate queue length estimations are possible, which can be used to adapt the algorithm to work more effectively to prevent long queues on the onramp. In that case, in the end cameras (or a combination of both) could be a better choice.

Another argument is that for a road manager the operational costs may be more important than the societal benefit for reducing vehicle hours. In that case, the costs will get a bigger weight which might lead to a choice for the cameras.

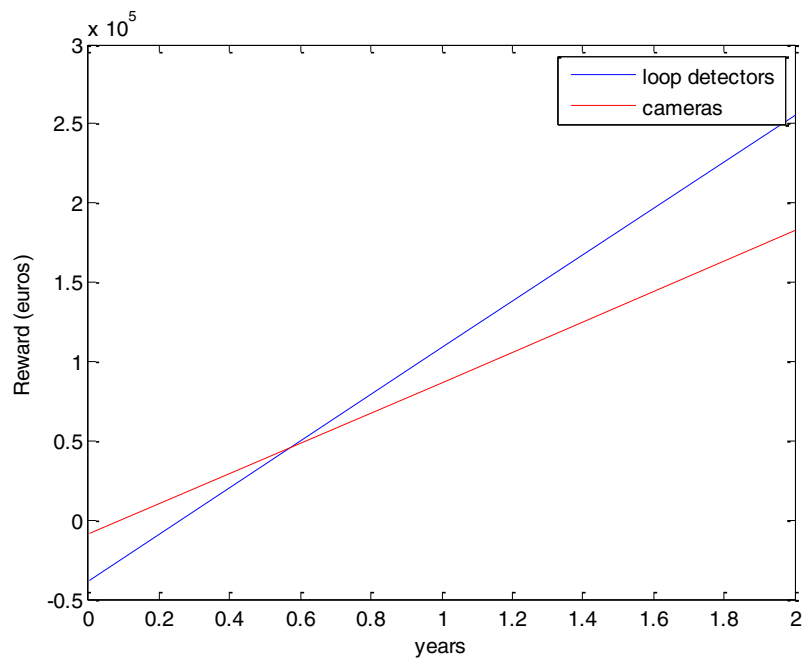

Fig. 6. Reward for loop detectors and cameras.

\section{CONCLUSIONS}

Linking traffic data quality to efficiency of traffic management is an unexplored field; while more and more traffic data are coming available, not much is known about the data quality required to reach the desired effectiveness of traffic management.

If the requirements for traffic data can be determined accurately for certain traffic management applications, this will give new possibilities for better traffic management:

It will lead to a better achievement of the traffic management goals with the same data, i.e. more efficient data use, and cost reduction, for example when less detailed/accurate data can be sufficient. In order to achieve this in the current world of traffic management practitioners, a change of view is needed: start with what you want to achieve instead of what data you have.

We've proposed an approach using information utility functions to study and quantify this relationship.

With a micro simulation study it has been shown that the accuracy of the input data has a large influence on the performance of a ramp metering system, and consequently on 
the network performance. However, even with an error of $50 \%$ the ramp metering system still improves the mean speed in the network compared to no ramp metering. The effect of inaccurate speed measurements has been shown to be larger than of inaccurate flow measurements. We've also quantified this relationship with a utility function based on a polynomial fit of the simulation results.

Finally, when loop detectors with a higher accuracy (but more expensive) are compared to cameras with a lower accuracy, it turns out that the difference in accuracy leads to an increase of the average network speed of $1.5 \mathrm{~km} / \mathrm{h}$ for the more accurate measurements of the loop detectors. Using an average value of time for the delay hours, already within one year it turns out to be more cost effective to use loop detectors instead of cameras. Note that these are not generalizable results, but a case study from one location which serves as an illustration of the type of analysis of the presented approach.

\section{FURTHER RESEARCH}

This research is part of a $\mathrm{PhD}$ research, which aims to address the problem of the relation between traffic data quality and traffic management/information in a broad perspective. Therefore, quality requirements will be established for several traffic management and information applications and situations. This will be done both for time critical applications such as ACC, medium time critical applications such as queue length estimation for urban control and less time critical applications such as routing and network-wide traffic management. In order to be able to generalize the results, a general framework will be proposed. The approach presented in this paper based on information utility functions will be extended for runtime optimisation using utility based reasoning. Also the type of errors that occur in reality on different types of traffic data will be investigated, as well as statistical relations between different types of errors.

\section{ACKNOWLEDGEMENT}

This research is supported jointly by TNO, Netherlands Organisation for Applied Scientific Research and TrafficQuest, a collaboration between Rijkswaterstaat, TNO and Delft University of Technology.

\section{REFERENCES}

Ahn, K., H. Rakha, D. Hill (2008). Data Quality White Paper. Publication FHWA-HOP-08-038. FHWA, U.S. Department of Transportation.

Ditzel, M., L. Kester, S. van den Broek and M. van Rijn (2013). Cross-layer Utility-based System Optimization. 16th International Conference on Information Fusion. Istanbul, Turkey.

Felici, E., O. Vroom (2012). Differentiation of data quality for Dynamic Traffic Management. 19th ITS World Congress, Vienna, Austria.

Foeken, E. van, L. Kester, M. van Iersel (2009). Real-time common awareness in communication constrained sensor systems. FUSION 2009: 118-125
Foeken, E. van, L. Kester. (2012). Adaptive team formation for shared situation awareness. FUSION 2012: 361-368

Gazis, D., and C. Knapp. On-line estimation of traffic densities from time-series of flow and speed. Transportation Science, 5, 1971, pp. 283-301.

Hoogendoorn, S., R. Landman, J. Van Kooten, M. Schreuder (2013). Integrated Network Management Amsterdam: Control approach and test results. IEEE Conference on Intelligent Transportation Systems, Proceedings, ITSC PP. 474 - 479 doi: 10.1109/ITSC.2013.6728276

IBM, http://www-01.ibm.com/software/data/bigdata/, accessed on April 12th, 2013.

Klijnhout, J.J. (1984). Motorway Control and Signalling: The Test of Time. Traffic Engineering and Control. Volume 25, No. 4, April 1984.

Klunder G.A., S.P. Hoogendoorn and H. Taale (2012). The relation between data quality and traffic management investigated for a simple route choice model. LATSIS Symposium 2012, 1st European Symposium on Quantitative Methods in Transportation Systems.

Lint, J.W.C. van, and S.P. Hoogendoorn (2007). The technical and economic benefits of data fusion for realtime monitoring of freeway traffic. World Congress of Transportation Research. Berkely, CA, USA, WCTRS.

Middelham, F., and H. Taale (2006). Ramp metering in the Netherlands: An overview. Control in Transportation Systems, Volume \# 11 | Part\# 1., pp. 267-272

Öörni, R., S. Innamaa, R. Kulmala, A. Kellermann, R. Ebner, and D. Newton (2010). Evaluation report for optimal data quality in selected European service cases. Deliverable 6 of QUANTIS - Quality Assessment and Assurance methodology for Traffic data and Information Services. Espoo, Finland.

Papageorgiou, M., H. Hadj-Salem and J.-M. Blosseville (1991) ALINEA: A local feedback control law for onramp metering. Transportation Research Record, 1320, pp. 58-64.

Saltelli, A., M. Ratto, T. Andres, F. Campolongo, J. Cariboni, D. Gatelli, M. Saisana, and S. Tarantola (2008). Global Sensitivity Analysis. The Primer, John Wiley \& Sons.

Tampère, C., F. Corman, W. Himpe (2011). Invloed van datakwaliteit op Dynamisch Verkeersmanagement. Report Katholieke Universiteit Leuven.

Treiber, M., and D. Helbinga (2002). Reconstructing the Spatio-Temporal Traffic Dynamics from Stationary Detector Data. Cooperative Transportation Dynamics 1 (3), 3.1-3.21

Viti, F., S. P. Hoogendoorn, L.H. Immers, C.M.J. Tampère and S. Hoogendoorn-Lanser (2008). National Data Warehouse: How The Netherlands is Creating a Reliable, Widespread and Accessible Data Bank for Traffic Information, Monitoring and Control of Road Networks. 87th Annual meeting of the Transportation Research Board.

Warffemius, P (2013). De maatschappelijke waarde van kortere en betrouwbaardere reistijden. Publication KiM13-A0. Ministry of Infrastructure and the Environment (Netherlands). ISBN: 978-90-8902-108-3. 